\title{
An application of six sigma for SMEs: A case study
}

\author{
Prabhakar Kaushik $^{a^{*}}$ and Sandeep Kumar
}

${ }^{a}$ Associate Professor, Mechanical Engineering Department, UIET, MD University, Rohtak, India

${ }^{b}$ M.Tech Scholar, Mechanical Engineering Department, UIET, MD University, Rohtak, India

\section{CH R O N I C L E A B S T RACT}

Article history:

Received: June 1, 2016

Received in revised format: No-

vember 16, 2016

Accepted: November 29, 2016

Available online:

December 5, 2016

Keywords:

Six sigma

Quality

Process improvements

Phases of implementation

Indian automobile sector

Quantitative tools

\begin{abstract}
Six Sigma is the concept of improving the quality by reducing process variations, making continuous improvements, reducing defect rates and improving the processes. Initially, the concept of Six Sigma focused on defect reduction, cost reduction and value addition. Fundamentally the basic idea of Six Sigma is to improve the process-capability and making the process more reliable along with reducing wastes within industries. Evaluation the implication of applying Six Sigma over the small and medium-sized enterprises is the main purpose of this research work taking a particular case of automobile industries. In the present work, DMAIC methodology of Six Sigma is used to a small seat slider lock nut manufacturing unit to reduce the play issue in K2 seat slider lock in automobile units by reducing defects inherent in the process. After applying Six Sigma methodology, it was found that manufacturing units could earn profits in terms of reducing the wastage as well as improving the quality standard of the product by controlling the play issue in seat slider lock nut mechanism. Result shows that with the application of Six Sigma, process, sigma level brought up to $5.53 \sigma$ from $1.59 \sigma$ by varying the seat slider lock nut dimensions/size.
\end{abstract}

\section{Introduction}

Six Sigma has been considered only to be meant for large scale manufacturing corporations/organizations and there was very silent documented evidences of its implementation in small scale manufacturing organizations (Kaushik et al., 2012). According to Abdelhamid (2003) and Biswas and Chowdhury (2016), it is nearly impossible to develop formal conceptual definition of Six Sigma because it is driven by the changing needs of the organization and consultant needs. It is evident from the fact that, "Six Sigma continues to draw tools and concepts such as Kano Analysis from marketing research, Change Management from organization behavior, Supply Chain Optimization from operations research and Hoshin Planning from Toyota". Companies may choose from the existing variations of this base definition while deploying Six Sigma in order to customize it for their situation (Plotkin, 1999). The deployment strategy may be different among them and also within, at various levels of organizations. During the period of later-half of the $19^{\text {th }}$ century, employee-driven improvements and incentive programs brought about positive changes in the industrial environment (Prasad, 2002). In the present work,

* Corresponding author.

E-mail address: parbhakarkaushik@yahoo.com (P. Kaushik) 
DMAIC methodology of Six Sigma is used to a small seat slider lock nut manufacturing unit to reduce the play issue in seat slider lock in automobile units by reducing defects inherent in the process.

\section{Problem Formulation}

Current study was conceded in a Small and Medium-sized Manufacturing Enterprises (SMEs) unit manufacturing automobile parts at Mohali in Punjab (India). The main product of the unit is seat slider and its components (Fig. 1 and Fig. 2). Slider lock is a vital sub assembly of seat slider assembly and its function is to eliminate the play issue in seat during sliding. The products of the unit are in the form of various seat slider lock nut used in automobile manufacturing. Slider shaft is the key component of automobile industries as it transfers the motion of the seat. The tolerance limit of the lock nut is 20.85-20.95 mm. Initial observation showed very high rejection of slider during working. So there was a great need to reduce rejection rate due to play in seat slider (Fig. 3). The adoption of project was accomplished by selecting Six Sigma DMAIC methodology for solving the project.

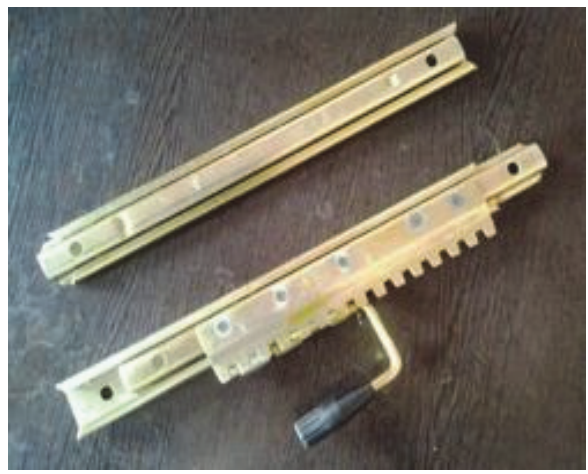

Fig. 1. Car Seat Slider

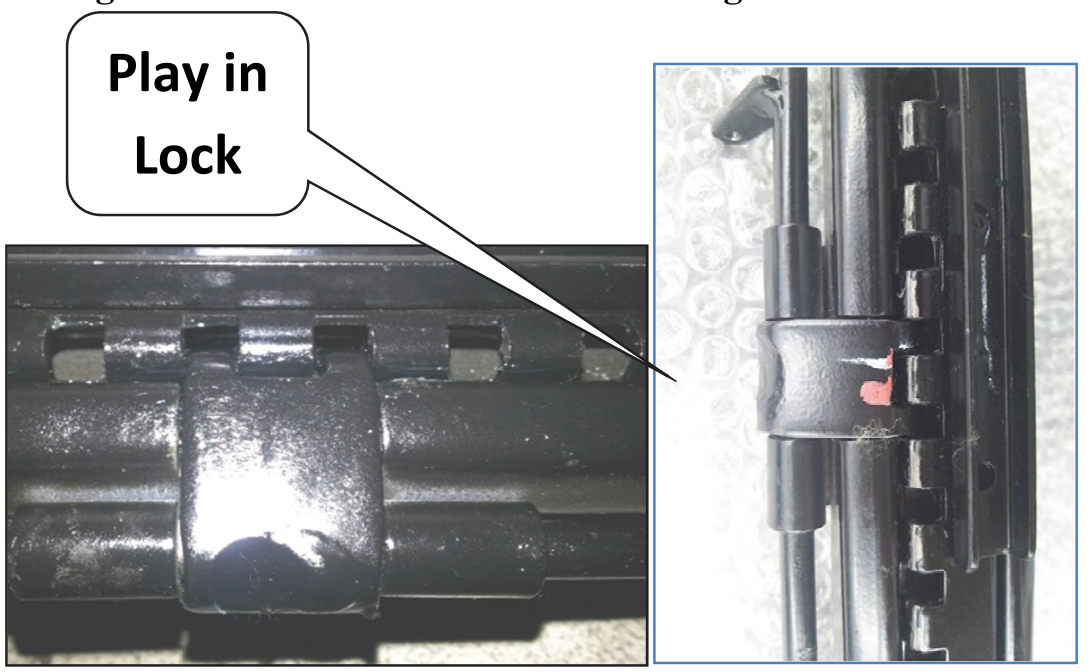

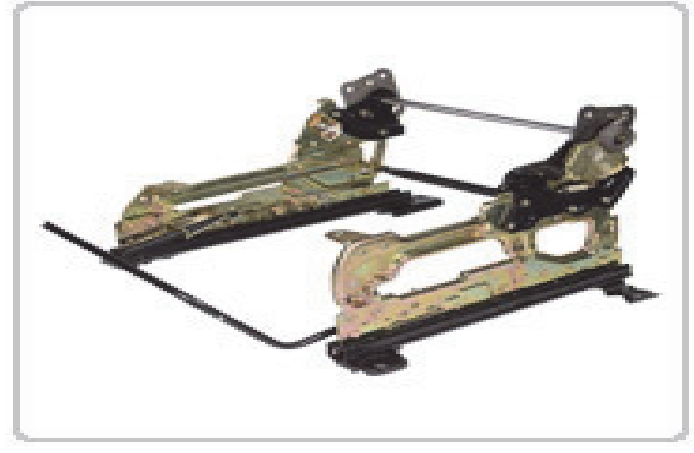

Fig. 2. Slider Recline

Fig. 3. Play in Lock 


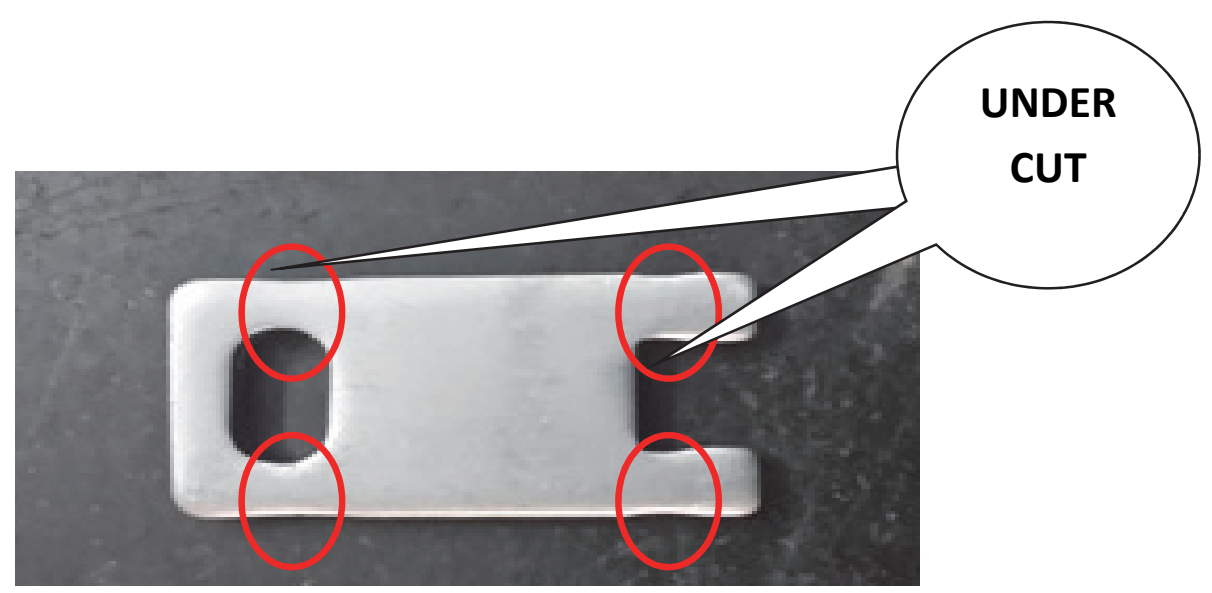

Fig. 4. Seat Slider Lock Nut

\section{Study and analysis of the play issue of seat slider lock rejection problem utilizing Six Sigma DMAIC methodology}

Seat slider lock nut is the key components of automobile as it transfers the motion of seat (Fig. 4). The tolerance limit of the lock nut slider is $20.85-20.95 \mathrm{~mm}$. The initial observation had been showing very high rejection rate for play issue in slider lock nut. The rejection rate of seat slider lock nut is 56396.75 ppm because of high variation in lock nut length. So, there was a great need to reduce to rejection rate of play issue in seat slider lock by reducing defect inherent in deferent processes. Six Sigma DMAIC methodology (Fig. 5) is used to solve play issue in slider lock rejection problem to achieve the quality level of 3.4 PPM from the present level of $56396.75 \mathrm{ppm}$.

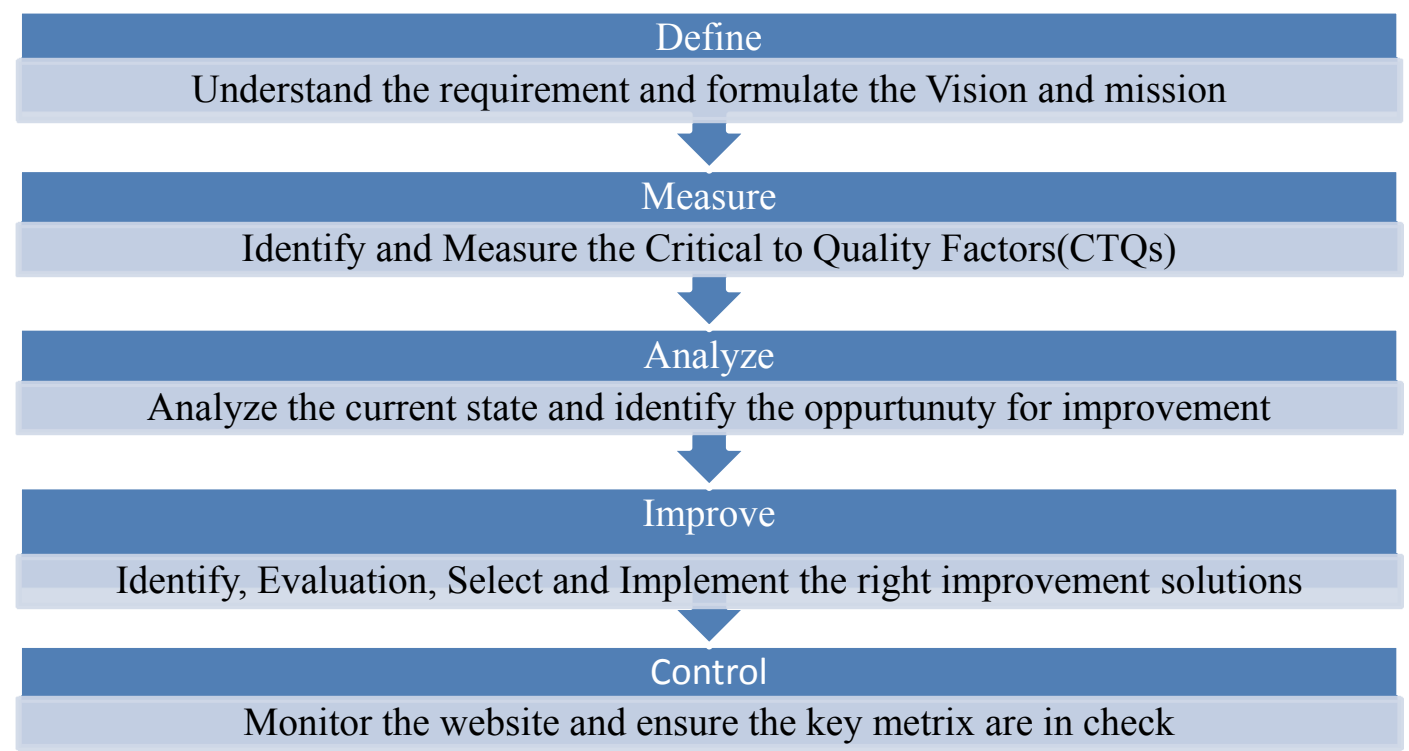

Fig. 5. DMAIC Methodology

In any organization project registration was the first step which shows the formal approval from the management to initiate the project, as it was never possible to implement suggestion and involve people without their backing (Wyper \& Harrison, 2000). With the help of the DMAIC methodology, problem 
of high rejection rate in play issue in seat slider lock nut was deeply studied which have been successfully implemented to achieve the sigma quality level of $5.53 \sigma$ from $1.59 \sigma$, as explained below.

\section{i) Define}

Define the problem to understand it easily (Raju, 2000).

Project Charter: The project title, problem statement, mission statement, project team composition and project milestones are mentioned in project charter.

Project Title: To eliminate the problem of play issue in seat slider due to space in between lock nut and seat slider assy.

Problem Statement: Currently in lock nut shaft, there is a problem of play issue in seat slider. 100\% rework has to be done before assembly.

Mission Statement: To eliminate the reworking of components (Savolainen, \& Haikonen, 2008).

\section{ii) Measure}

For the measurement of the lock nut dimension we studied Measurement System Analysis (MSA) which includes the Gauge repeatability and Reproducibility (Gauge R\&R) to ensure for the statistical sound (Kaushik \& Khanduja, 2008). Gauge R\&R studies show how much of the variation in the measurement in the observation process is needed. Two observers are needed to perform this experiment, one operator on the line and other the invigilator. Total sample was 100 and two reading were taken so total reading have 200. Micrometer was used for this experiment as a gauge. Result from the gauge repeatability and reproducibility (Gauge R\&R) was 29.73 percent which was less than the 30 percent for the study variation percent was putting 0.00 percent indicating that the Measurement System was correct.

\section{iii) Analysis}

\section{- Process Capability Analysis}

This process was done to determine actual state of this process (Snee, 2004). 100 samples were drawn in two groups for rational sub-grouping. With the help of the Minitab software process capability analysis curve was drawn for seat slider lock nut shown in Fig. 6. Its Z-bench sigma level value was 1.59 and DPMO level was 56396.75 which shows significant amount of improvement opportunities required in this process.

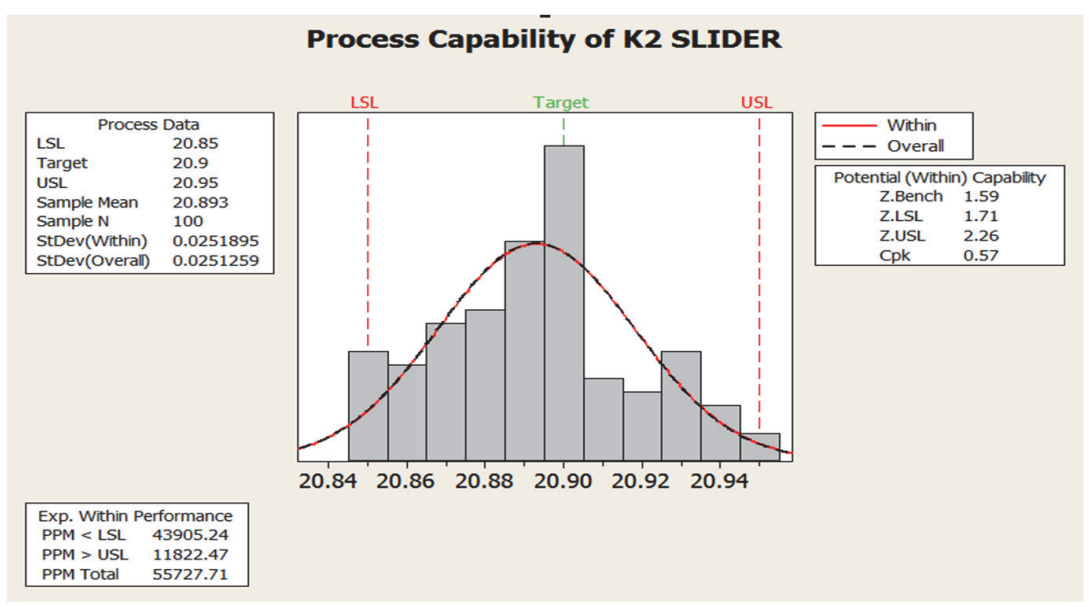

Fig. 6. Process Capability Analysis of Lock Nut Slider 


\section{- Fish- Bone Diagram}

After knowing all issues regarding to seat slider lock nut like DPMO level, process capability analysis and sigma level, we have found out the main cause of rejection of the sear slider lock nut. A fishbone diagram was drawn (Fig. 7) to explain all causes of seat slider lock nut rejection using the expert experience and brainstorming sessions.

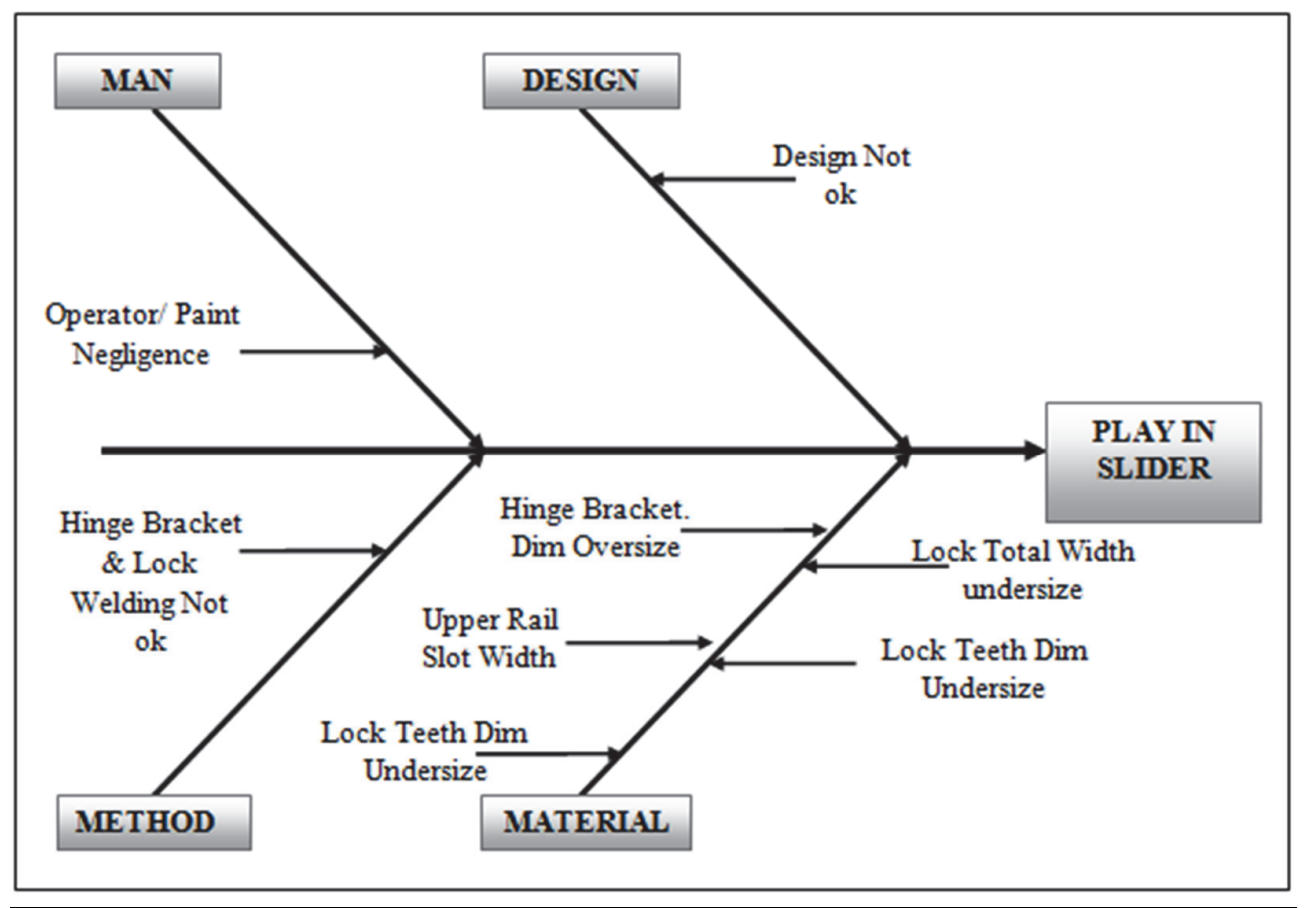

Fig. 7. Fishbone Diagram

\section{- Two Sample-T Test}

Important factors from the two sample-t test were taken for study identified from fishbone diagram. A test of two sample $t$ test was done for the first case for operator skill (unskilled and skilled) taking 50 sample size each for both skilled and unskilled.

\section{Table 1}

Two Sample $t$ test for operator skill

\begin{tabular}{ccccc}
\hline Operator & $\mathrm{N}$ & Mean & SD & SE mean \\
\hline 1 & 50 & 20.8992 & 0.0239 & 0.0034 \\
2 & 50 & 20.9022 & 0.0187 & 0.0026 \\
\hline
\end{tabular}

Difference $=\mathrm{mu}($ Operator 1$)-\mathrm{mu}($ Operator 2),

Estimate for difference: -0.00300

95\% upper bound for difference: 0.00412 ,

$\mathrm{T}$-Test of difference $=0(\mathrm{vs}<)$ : T-Value $=-0.70 \mathrm{P}-$ Value $=0.243 \mathrm{DF}=92$

\section{Table 2}

Two-sample t-test: Component Alignment (Gap Maintain) Two-sample t-test: Lock nut width

\begin{tabular}{ccccc}
\hline Operator & $\mathrm{N}$ & Mean & SD & SE mean \\
\hline Initial Reading & 50 & 20.8942 & 0.0256 & 0.0036 \\
Final Reading & 50 & 20.9022 & 0.0187 & 0.0026 \\
\hline
\end{tabular}

Difference $=\mathrm{mu}$ (Initial Reading) - mu (Final Reading),

Estimate for difference: -0.00800

$95 \%$ upper bound for difference: -0.00055 ,

T-Test of difference $=0(\mathrm{vs}<)$ : T-Value $=-1.78, \mathrm{P}-$ Value $=0.039, \mathrm{DF}=89$ 
Using Minitab, the Two Sample T-Test shows that as the p-value for component alignment comes out to be less than 0.05 therefore lock nut width can be a factor for seat slider lock shaft rejection.

\section{iv) Improve}

In improve phase, the counter measures of these causes is developed (Srinivasan et al., 2016) and it has been applied to reduce the play issue in seat slider and productivity improvement. Root cause and its remedy has shown it and the factors that comes out to be the key reasons for the high rejection of seat slider lock shaft are component alignment. Suitable counter measures were implemented. Lock blank modified by increasing the width (at areas "A" \& "B") to maintain dimension of Tip of Lock, Root of Lock, Root of blank. The width of lock has been increased in such a way that it would compensate the increase in width during bending operation and maintain $20.8 \mathrm{~mm}$ dimension.

\section{Before}

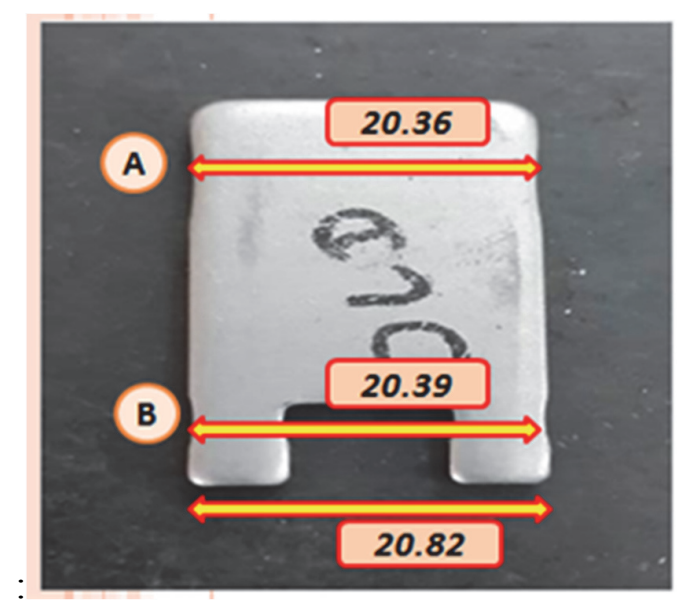

After

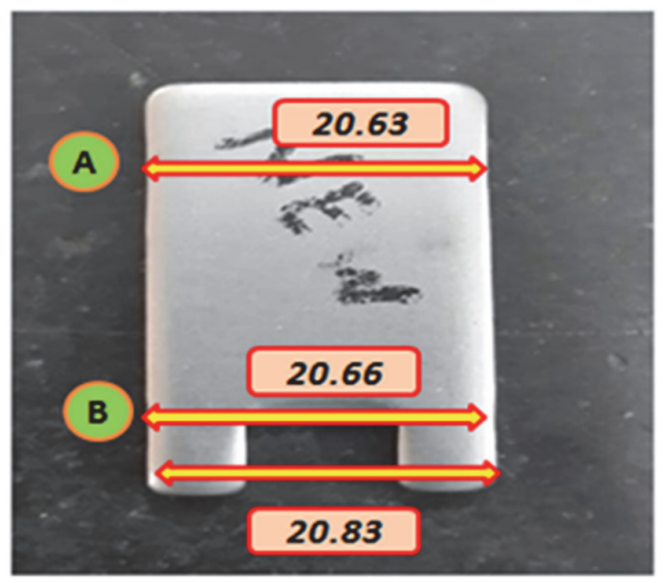

Fig. 8. Lock Nut Dimension Before and After

\section{v) Control}

$\mathrm{X}$ bar/R Control Chart was drawn by taking 100 sample size after changing the dimension of the lock nut size to visualize the presence of assignable cause (Fig. 9). Final result shows that there was no play issue in seat slider after changing the dimension as per recommendation in improve phase and it was smoothly slide ensured optimized results.

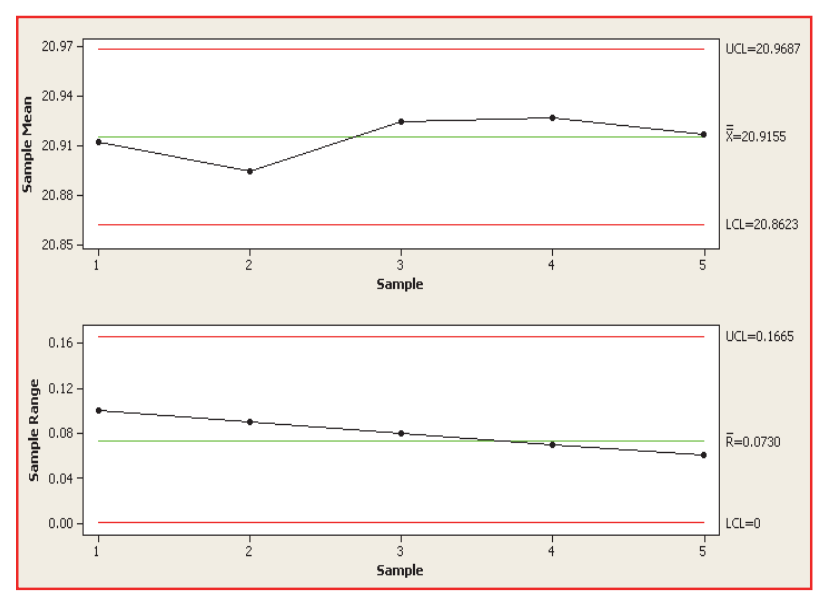

Fig. 9. Control Chart 


\section{Result appraisal and conclusions}

Various researchers have argued that Six Sigma as a methodology is a very effective management tool that covers the $5 \mathrm{Ms}$ (machines, men, money, management and materials) within the larger framework of operations management. Six Sigma is a revolutionary tool which can be broadly used in future for saving money, time and reducing significant amount of wastages in every field (Thomas \& Barton, 2006). Application of project recommendation brought up the sigma level up to 5.53 from 1.59 (Fig. 10) which is equivalent to monetary saving of money per annum and is substantial for a small organization.

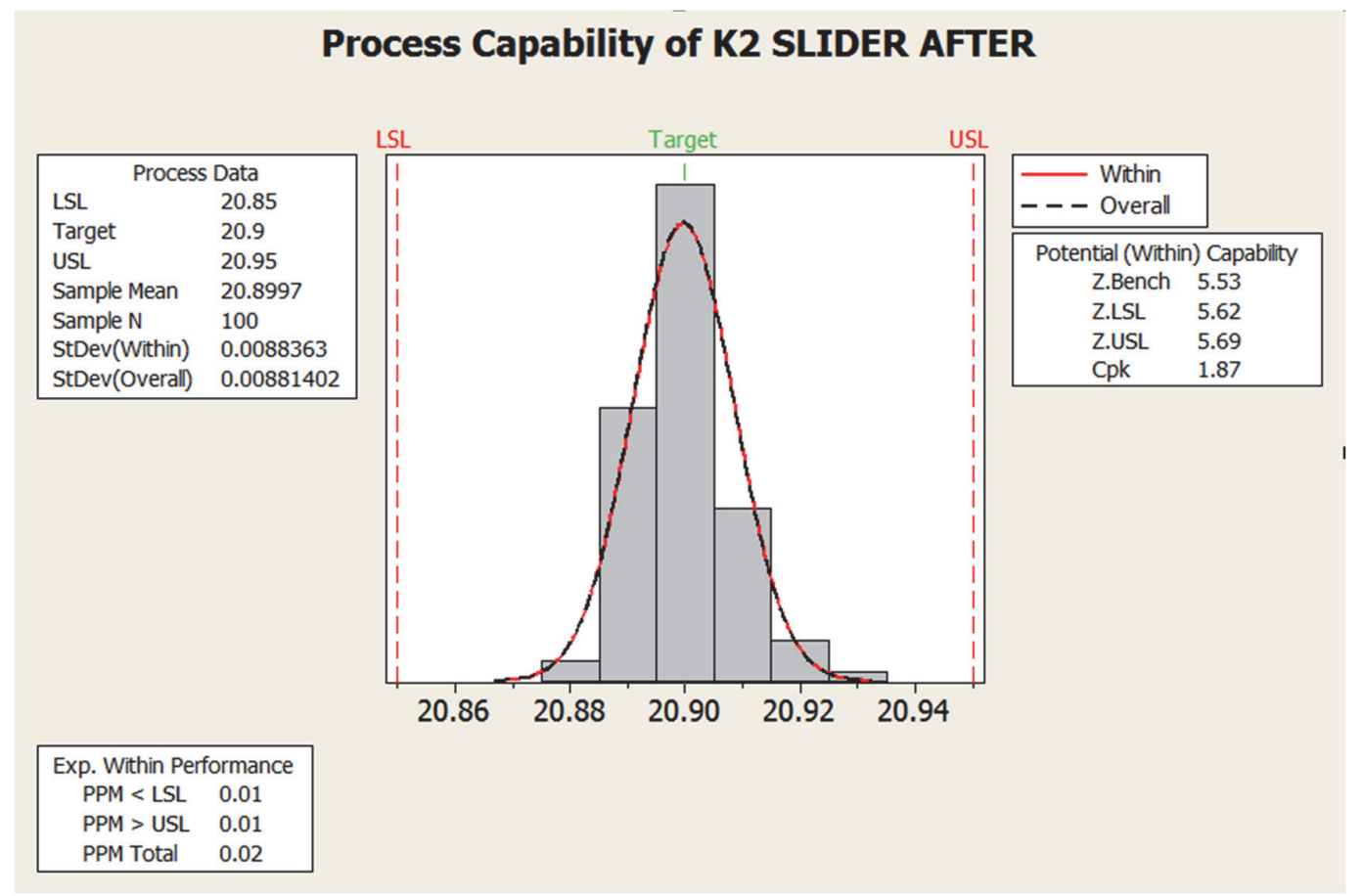

Fig. 10. Process Capability of Lock Nut Slider after Implementing DMAIC

\section{References}

Abdelhamid, T.S. (2003). Six Sigma in lean construction systems: opportunities and challenges. Proceedings of the $11^{\text {th }}$ annual conference for lean constructions, July 22-24, Blacksburg, Virginia, 6583.

Biswas, S., \& Chowdhury, B. (2016). Industrial Applications and Practices of Six Sigma - A Literature Review. International Advanced Research Journal in Science, Engineering and Technology, 3(3), 176-179.

Kaushik, P., Khanduja, D., Mittal, K., \& Jaglan, P. (2012). A case study: Application of Six Sigma methodology in a small and medium-sized manufacturing enterprise. The TQM Journal, 24(1), 416.

Kaushik, P., \& Khanduja, D. (2008). DM make up water reduction in thermal power plants using Six Sigma DMAIC methodology. Journal of Scientific and Industrial Research, 67(1), 36-42.

Plotkin, H. (1999). Six Sigma: What it is and how to use it. Harvard management update, June edition, 06-07.

Prasad, J. (2002). Six Sigma in bulb manufacturing. Productivity journal, 43(2), 192-195. 
Raju, R. (2000). Reducing axle rejection using problem solving tools-a case study. Industrial Engineering Journal, 29(2), 24-29.

Savolainen, T., \& Haikonen, A. (2007). Dynamics of organizational learning and continuous improvement in Six Sigma implementation. The TQM Magazine, 19(1), 6-17.

Snee, R. D. (2004). Six-Sigma: the evolution of 100 years of business improvement methodology. International Journal of Six Sigma and Competitive Advantage, 1(1), 4-20.

Srinivasan, K., Muthu, S., Devadasan, S. R., \& Sugumaran, C. (2016). Six Sigma through DMAIC phases: a literature review. International Journal of Productivity and Quality Management, 17(2), 236-257.

Thomas, A., \& Barton, R. (2006). Developing an SME based six sigma strategy. Journal of Manufacturing Technology Management, 17(4), 417-434.

Wyper, B., \& Harrison, A. (2000). Deployment of Six Sigma methodology in human resource function: a case study. Total Quality Management, 11(4-6), 720-727.

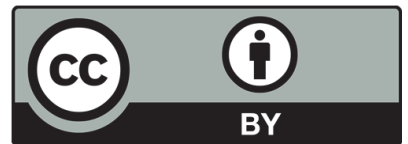

(C) 2017 by the authors; licensee Growing Science, Canada. This is an open access article distributed under the terms and conditions of the Creative Commons Attribution (CC-BY) license (http://creativecommons.org/licenses/by/4.0/). 